\section{Technologies for the development of West Nile virus vaccines}

\author{
Sebastian Ulbert*,1 \& Sofia E Magnusson²
}

\begin{abstract}
West Nile virus (WNV), an emerging mosquito-borne and zoonotic flavivirus, continues to spread worldwide and represents a major problem for human and veterinary medicine. In recent years, severe outbreaks were observed in the USA and Europe with neighboring countries, and the virus is considered to be endemic in an increasing number of areas. Although most infections remain asymptomatic, WNV can cause severe, even fatal, neurological disease, which affects mostly the elderly and immunocompromised individuals. Several vaccines have been licensed in the veterinary sector, but no human vaccine is available today. This review summarizes recent strategies that are being followed to develop WNV vaccines with emphasis on technologies suitable for the use in humans.
\end{abstract}

\section{West Nile virus}

First described in 1937 in the West Nile district in Uganda, West Nile virus (WNV) is today one of the most widely spread arthropod-transmitted pathogens. WNV belongs to the Flaviviridae family, which also includes the human pathogens yellow fever virus, tick-borne encephalitis virus, Japanese encephalitis virus (JEV) and dengue virus [1]. Within the flaviviruses, WNV is grouped into the JEV serocomplex, being highly similar to JEV, Usutu and St Louis encephalitis viruses. WNV infects birds and is transmitted by a large range of different mosquito species. The presence of suitable insect vectors in almost all parts of the world enables the virus to efficiently invade novel territories. Besides birds, only few animal species (alligators and some frog species) are known to function as hosts that can re-infect mosquitoes. Nevertheless, the insect vectors can transmit WNV to a variety of other animals, including mammals, although these represent dead-end hosts for the virus. In humans, most infections remain asymptomatic and approximately $20 \%$ of the cases result in mild disease associated with flu-like symptoms. In approximately $1 \%$ of the infected individuals, however, severe symptoms occur ranging from high fever to neurological disease including flaccid paralysis, meningitis and encephalitis. Aged or immunocompromised individuals are at highest risk for developing these severe forms of West Nile disease, which may even be fatal in up to $10 \%$ of the severe cases $[2,3]$.

Only sporadic outbreaks of WNV among humans or horses were observed between the 1940s and 1980s in Africa, Asia and some Mediterranean countries of Europe. By contrast, in the 1990s, the virus started to appear more frequently, and, in 1999, the first cases were observed in the USA [4]. In the following years, WNV rapidly spread over entire North America and has reached several Latin and South American countries. Total case numbers of severe infections in the USA

'Department of Immunology, Fraunhofer Institute for Cell Therapy \& Immunology, Perlickstrasse 1, 04103 Leipzig, Germany

${ }^{2}$ Novavax AB, Kungsgatan 109, 75318, Uppsala, Sweden

*Author for correspondence: Tel.: +49 341 355362106; sebastian.ulbert@izi.fraunhofer.de

\section{KEYWORDS}

- animal models $\bullet$ emerging virus $\bullet$ protective immune responses $\bullet$ vaccine development $\bullet$ West Nile Virus 
until today exceed 37,000 , including more than 1500 deaths. In addition, the WNV epidemic was accompanied by massive mortality in birds and horses [5].

Over the last 6 years, outbreaks are also increasing in number in Europe. WNV cases among humans are now seen yearly in some areas of Italy, Hungary, Greece, Russia and several other southern and eastern European countries. Hence, the virus is considered to be endemic in many areas of Europe.

Several genetic lineages of WNV exist, and most circulating strains can be attributed to the two major lineages 1 and 2, which are approximately $94 \%$ identical on the amino acid level [6]. Until very recently, lineage 1 viruses were associated with severe outbreaks among humans and horses, whereas lineage 2 was considered to be much less pathogenic. However, the situation has changed in recent years. Following the identification of more virulent lineage 2 strains in South Africa [7], major outbreaks among humans in countries such as Greece or Russia have been caused by WNV from lineage $2[8,9]$. Even co-circulation of lineage 1 and 2 strains has been demonstrated $[10,11]$. The mechanisms underlying this diversification and the spread of WNV within Europe still need to be fully elucidated.

WNV is an enveloped virus with a ssRNA genome of approximately $11 \mathrm{~kb}$ in positive orientation. The viral genome encodes for seven nonstructural proteins and the three structural proteins $\mathrm{C}$ (capsid protein), prM (membrane protein) and $\mathrm{E}$ (envelope protein). The capsid, containing the RNA, is made of multiple copies of the $\mathrm{C}$ protein. The prM and $\mathrm{E}$ proteins reside in the lipid envelope of WNV. In the mature virus, the pr precursor peptide is split from the $\mathrm{M}$ protein, which has important roles in stabilizing the correct conformation of $\mathrm{E}$. The $\mathrm{E}$ protein fulfils critical functions in the viral life cycle, such as receptor binding and the escape from vesicles after the endocytosis into the host cell [12]. Consequently, it is the major target for protective immune responses (see below). The nonstructural proteins of WNV remain intracellular and mediate the RNA replication and virus assembly, only nonstructural protein 1 (NS1) is associated with the surface of infected cells and is also secreted [13,14]. Antibodies against NS1 are able to protect mice from WNV infection [15]. In addition to roles in virus structure and assembly, several WNV proteins also interfere with specific pathways of the innate immune response of the host and thereby help the virus to successfully establish an infection [16-18].

\section{Vaccine-mediated protection from WNV}

Vaccines to protect humans against a number of flaviviruses such as tick-borne encephalitis virus, JEV or YF, are available, hence the principles underlying these vaccines are obvious candidates for developing vaccines for WNV. This is especially the case for JEV, which is the only flavivirus within the JEV serocomplex that is targeted by commercially available vaccines for humans, and immunization is performed using inactivated, attenuated or recombinant chimeric viruses $[19,20]$. However, with WNV aged and immunocompromised individuals are at highest risk for severe infections and, therefore, the major target groups for vaccines. Hence, not all technologies of existing human flavivirus or veterinary WNV vaccines are suitable for the development into a human WNV vaccine.

WNV only sporadically caused outbreaks before the 1990s, and therefore the virus was previously not in focus of intense vaccine research efforts. However, after the introduction of WNV into the USA in 1999, the prevention of virus infection became an important topic in both academic research and industry. Due to the observed high mortality among WNV-infected horses, the development of a formaldehyde-inactivated whole-virus vaccine for veterinary use was initiated, and this vaccine was successively licensed in 2003 [21]. In addition, a canarypox virus-based vaccine expressing the prM and E proteins of WNV became available in 2004 [22]. Immunization of horses led to a marked decrease of severe WNV disease in the following years [23], demonstrating the effectiveness of these vaccines. In addition, they were also used to protect other animal species, especially birds [24]. Nevertheless, all veterinary WNV vaccines require an annual booster immunization in order to remain protective. Hence, there is clearly need for WNV vaccine technologies delivering a longer-lasting immunity, especially for the use in humans as no human vaccine exists to date.

Although the exact levels of immunity correlating to protection from WNV disease have not been defined yet, the development and testing of different immunization strategies 
have already yielded valuable insight into the immune mechanisms underlying vaccinemediated protection. Both the innate and the adaptive immune system are active against the virus and perturbation of either of them can lead to high susceptibility towards an infection. The immune response against a WNV infection has been studied primarily in different rodent models and has been reviewed in detail elsewhere [25-28].

Based on a substantial amount of data, it is currently well accepted that the humoral immune response is a key player in vaccinemediated protection: B-cell-deficient mice can be protected from WNV disease by the passive transfer of antibodies from WNV-immune animals and the level of neutralizing antibodies correlates with protection $[29,30]$. In addition, potent neutralizing antibodies have been identified and evaluated in human clinical trials [31,32]. An investigation of the immunological memory response to WNV revealed that antibodies derived from either vaccine-induced memory $\mathrm{B}$ cells or long-lived plasma cells are protective. However, protective antibodies against a heterologous WNV strain are mostly produced by memory B cells, which is especially relevant for crossprotection against the different circulating WNV lineages [33].

WNV infection in mice antibodies of the IgG2 isotypes have shown to have enhanced effector functions, due to increased complement binding to their Fc regions, and this function of WNV-specific IgG2 helps to reduce the total amount of antibodies needed for neutralization of WNV and thereby enhances antibody-mediated protection against WNV in mice [34]. In contrast to antibodies, $\mathrm{CD}^{+}{ }^{+} \mathrm{T}$-cell responses seem to play a more supportive role in vaccinemediated protection, especially after repeated rounds of immunization [35].

Most animals and humans infected with WNV generate a robust antibody response against the $\mathrm{E}$ protein $[36,37]$. This protein represents the major target for protective WNV antibodies, although protective and neutralizing antibodies have also been described against the NS1 and M proteins, respectively [15,38]. Consequently, almost all current approaches for WNV subunit vaccines represent methods to deliver the E protein. Within E, the domain DIII, which is involved in receptor binding, harbors important epitopes for effective neutralization of the virus [39-41]. Immunization with only recombinant domain DIII was sufficient to protect animals from WNV [42,43]. However, in humans, the humoral immune response to a WNV infection is skewed towards non-neutralizing epitopes in other parts of the E protein, mainly clustering in or near the fusion loop [41,44]. Whether mutant forms of the E protein, which lack the immune-dominant non-neutralizing epitopes at the fusion loop [45], would have a stronger protective effect in humans remains to be tested.

The availability of several suitable animal models, the possibility to grow WNV well in cell culture, the use of reverse genetics and the focus on the E protein as the major protective antigen have led to a variety of different approaches for WNV vaccination. These include the latest developments in subunit immunization such as vector- or DNA-based technologies.

\section{Vaccines containing complete WNV particles}

Several formaldehyde-inactivated whole-virus vaccines are available for veterinary use. They are based on different WNV strains and are widely used to immunize horses. As formaldehyde treatment negatively affects the antigenic structures of the pathogen [46,47], alternative inactivation procedures are under development, in particular when addressing the use in humans. Amanna et al. [48] established an inactivation method based on the incubation of the low-pathogenic Kunjin strain of WNV with hydrogen peroxide, which leads to the formation of oxygen radicals. This technology mainly destroys the viral RNA genome but causes much less damage to protein antigens. A WNV vaccine produced by this method completely protected mice against infection with WNV and elicited higher levels of anti-WNV antibodies as compared with a commercial, formaldehydeinactivated one. In addition, the protective antibody levels remained high for more than 280 days [48].

The yellow fever vaccine based on the 17D strain represents an example for the attenuation of a flavivirus, and its successful use as a vaccine, but only few vaccine approaches relying on a classically attenuated WNV have been described [49]. By contrast, attenuation of the virus by using reverse genetics (i.e., inserting mutations at defined positions in the genome) is seen as a promising alternative that could provide clear benefits regarding safety issues. 
Several mutations in the coding sequences for the capsid protein lead to the production of defective viruses that are still immunogenic enough to induce protection [50]. Given the target population for WNV vaccines, technologies relying on classical attenuation or single point mutations need to be carefully checked for safety issues that might arise through reversion of mutations.

By deleting the sequence for the capsid and supplying this information in a helper cell line, Widman et al. [51] generated virus particles that were immunogenic but lacked the capacity to successfully replicate in the infected cell. The vaccine generated by this technology, called RepliVAX, proved to be protective in several animal models including nonhuman primates.

In another strategy by Whiteman et al. [52], WNV viruses (derived from strain New York 1999 [NY99]) with mutations in the glycosylation sites for the E and NS1 proteins were produced as potential vaccine candidates. Mice infected with these mutant viruses showed no viremia in the blood but had high titers of neutralizing antibodies [52]. Furthermore, the mice were protected against lethal challenge with the wild-type NY99 strain.

\section{Recombinant protein vaccines}

WNV protein subunit vaccine candidates intended for human use have been evaluated preclinically (both in rodents and nonhuman primates) and in Phase I clinical trials. In most approaches, different forms of the ectodomain of the $\mathrm{E}$ protein have been evaluated as vaccine antigens, including the isolated domain DIII. The C-terminal transmembrane region of $\mathrm{E}$ is usually excluded from these antigens, as it causes difficulties in the expression of the protein. In addition, as subunit vaccine antigens lack inherent immune-stimulatory properties such as Toll-like receptor (TLR) ligands or pathogen-associated molecular patterns, the $\mathrm{E}$ protein vaccine candidates have for the most part been formulated with various adjuvants to ensure that a broad and effective humoral and cellular immune response is elicited.

Wang et al. [53] expressed the E ectodomain (amino acid residues 1-406) as a recombinant thioredoxin fusion protein in Escherichia coli and formulated the recombinant protein with Freund's complete adjuvant (FCA) before immunizing mice. The vaccine proved to be protective in a WNV challenge model and human serum antibodies from previously WNV-infected individuals recognized the recombinant E protein used. However, a rather high antigen dose $(20 \mu \mathrm{g}$ per mouse and immunization) was required even though the protein was adjuvanted with the potent adjuvant FCA, which, however, is not allowed for human use [53].

The WNV E ectodomain has also been expressed in insect cell systems - that is, Drosophila S2 cells and in Spodoptera frugiperda expresSF+ cells via baculovirus infection [5456]. These proteins have been comprehensively evaluated as WNV vaccine antigens [56-58]. Ledizet et al. [54] and Bonafé et al. [56] describe studies with an insect cell-derived antigen $\left(\mathrm{rWNV}-\mathrm{E}_{\mathrm{T}}\right)$, formulated with either Alum or FCA, which was evaluated in mice, hamsters and horses. Both formulations were protective against lethal WNV challenge in mice and hamster models. When horses already vaccinated with a commercially available inactivated horse WNV vaccine, named Equip WNV, were boosted with Alum-adjuvanted rWNV-E ${ }_{\mathrm{T}}$ they produced higher titers of WNV-neutralizing antibodies compared with horses boosted with the inactivated vaccine [56]. In these studies, only total $\operatorname{IgG}$ was measured, the quality of the antigen-specific T-cell response was not investigated and is thus unknown. In a similar approach, a recombinant $\mathrm{E}$ ectodomain protein (E80) and a recombinant version of the NS1 protein were produced in the Drosophila S2 expression system [55]. Both proteins were evaluated in mice and hamsters as a combination or as single protein vaccines formulated with the saponin-based adjuvant ISCOMATRIX ${ }^{\circledR}$ (CSL Ltd, Melbourne, Australia) [55,57] with antigen doses ranging from 0.3 to $10 \mu \mathrm{g}$. Vaccination with the E80 alone, NS1 alone or E80 plus NS1 led to high neutralizing antibody titers and good antigen-specific cellular responses in mice, as measured by antigen-specific proliferation and cytokine production [55]. The antibody response was biased towards $\operatorname{IgG} 1$ as the $\operatorname{IgG} 2 \mathrm{a}$ titers were lower than the IgG1 titer in all vaccine groups. Moreover, in a hamster WNV challenge model with a mortality rate of $50 \%$, vaccination with the E80 protein, adjuvanted with ISCOMATRIX, led to $100 \%$ protection whereas vaccination with NS1 protein was only partially protective [57]. The rodent study was followed by a WNV efficacy study of rhesus macaques vaccinated with $5 \mu \mathrm{g}$ E80 adjuvanted 
with GPI-0100, an adjuvant comprised of semisynthetic quillaja saponins [58]. All vaccinated monkeys developed WNV-neutralizing antibodies and were protected from WNV infection as no viremia was detected in the blood 10 days after the infection. In 2009, a Phase I clinical trial was performed in healthy adults using the $\mathrm{E} 80$ vaccine antigen adjuvanted with Alhydrogel ${ }^{\circledR}$ (Invivogen, Toulouse, France) [59].

More recently, another E. coli-expressed WNV E protein was extensively evaluated preclinically as a possible human WNV vaccine [60]. The protein was produced by expressing the $\mathrm{E}$ protein ectodomain (amino acid residues 1-404) of the NY99 strain in E. coli, and purifying it after oxidative refolding [41]. To ensure an effective immune response with both humoral and cellular immunity the protein was formulated with the particulate Quillaja saponaria saponin-based adjuvant Matrix-MTM (Novavax AB, Uppsala, Sweden). Adjuvant Matrix-M can induce a broad immune response, comprising Th1 and Th2 responses, compared with using antigen alone or antigen mixed with other adjuvants $[61,62]$. The adjuvant has been evaluated in several Phase I clinical trials with very good safety data [63]. The E protein as a WNV vaccine antigen was evaluated using different antigen doses $(0.5-10 \mu \mathrm{g} / \mathrm{mouse})$ formulated with different adjuvant concentrations [60]. This vaccine composition induced higher antigen-specific IgG1 and IgG2a titers, as well as neutralizing WNV antibodies, when compared with the same antigen formulated with the Th2-prone adjuvant Alum. Additionally, the E proteinspecific cellular immune response was increased in comparison with the Alum-adjuvanted antigen: vaccinated mice had significantly higher levels of Th1 and Th2 cytokines from ex vivo antigen-stimulated splenocytes. Moreover, the addition of Matrix-M made it possible to reduce the antigen dose six-times while maintaining comparable humoral responses. Two separate WNV challenges, one using a homologous WNV strain (lineage 1, strain ITA-09) to the vaccine antigen and another using a heterologous WNV lineage 2 (strain AUT-08), revealed that mice were completely protected from WNV disease after vaccination with one or two doses of the vaccine.

As mentioned earlier, the DIII part of the E protein has also been evaluated preclinically in mice. In the paper by Chu et al. [64], immunization with WNV DIII, expressed in E. coli, gave rise to both $\operatorname{IgG} 1$ and $\operatorname{IgG} 2 \mathrm{a}$ anti-DIII antibodies and a strong Th1 cellular response. These antibodies protected young mice from cerebral WNV infection. The immune response induced by WNV DIII was further enhanced using CpG DNA as an adjuvant. However, to induce the reported immune responses, the vaccine was intraperitoneally injected at a very high antigen dose $(100 \mu \mathrm{g}$ recombinant DIII) at three time points with 1 week intervals, which makes it hard or practically impossible to translate into future human use. This observation corresponds to a detailed analysis by Zlatkovic et al. [65], who demonstrated that DIII alone was much less effective in inducing WNV-neutralizing antibodies than the E ectodomain or inactivated complete virions. The recombinant protein probably exposes domains that are cryptic in the virion and, thereby, focuses the antibody response away from key neutralizing determinants [65]. In another approach, the DIII domain was expressed in $E$. coli as a recombinant protein fused to a modified version of the bacterial protein flagellin (STF2 $\Delta$ ) [66]. This confers the DIII domain with an inherent adjuvant effect as flagellin binds to TLR5, which is expressed by important innate immune cells such as neutrophils, monocytes and dendritic cells. When tested in mice, this fusion protein gave rise to WNV neutralizing antibodies and protected mice against a WNV challenge. When the fusion protein was injected in TLR5-deficient mice the DIII-specific antibody titers dropped drastically, which implies that the TLR5 activation of the innate immune system, and also the probably enhanced uptake of DIII by phagocytes, was necessary for protection.

\section{DNA vaccines}

As an alternative approach to using recombinant proteins or whole viruses as vaccines, the antigens can be delivered in form of their DNA sequences. This technology has the advantage that the antigens are made by the cells of the vaccinated individual itself, engaging both the MHC class 1 and class 2 pathways of antigen presentation and thereby mimicking a viral infection. As the nucleic acid sequences can easily be changed, this technology is especially suitable for the rapid adaptation of a vaccine towards newly emerging variants of the pathogen. Although immunization with nucleic acids is the topic of several clinical trials in humans, 
all licensed DNA vaccines so far are in the veterinary sector, including one for WNV. This particular vaccine co-expresses the prM and the E proteins, which in cell culture leads to the formation of subviral particles that closely resemble the complete WNV virion [67]. This strategy to combine the expression of $\mathrm{E}$ protein with the expression of prM is also used in other vaccine technologies (see below). Although protective effects were shown in mice, horses and birds, the vaccine was not made available [24,68]. A similar DNA plasmid has also been tested in humans in a Phase I clinical trial, demonstrating its potential to elicit WNV-neutralizing antibodies [69,70]. Other recent experimental DNA vaccines that showed protection in the mouse model used the ectodomain of the $\mathrm{E}$ protein either fused to a secretory signal sequence or to parts of the complement protein C3d [71,72]. A more complex system that yields single-round infectious WNV particles similar to the before mentioned RepliVAX technology was constructed by Chang et al. [73]. A single DNA plasmid generates two different RNAs, which allow the formation of structurally intact WNV particles. As these virions do not contain functional RNA for the capsid protein, new viral particles cannot be assembled in the cell after a single round of infection.

The major general drawback of DNA vaccination is its relatively low immunogenicity likely due to the inefficient delivery of the plasmids into the cell nucleus [74]. As a consequence, in animals larger than mice, high (i.e., $\mathrm{mg}$ ) quantities of DNA need to be applied in order to elicit a significant immune response. Therefore, despite its clearly demonstrated qualities in immune stimulation, safety and stability, DNA vaccination still awaits an efficient delivery technique. The gold standard technology today is in vivo electroporation, which transfers DNA into the cell by applying short electric pulses to the site of injection [75]. However, this technology is still very sophisticated (with a complex apparatus required) and quite painful, which makes its use in mass vaccination scenarios still unlikely. Alternative strategies focus on the development of nanoparticles that carry the DNA plasmid across the cellular and nuclear membranes or on the delivery via micro-needles [76]. Recently, mannosylated polyethylenimine was used to complex a WNV DNA vaccine plasmid into nanoparticles that were then administered to mice either intramuscularly or directly onto the skin [77]. With the mannose moiety the resulting DNA nanocomplexes are able to bind to the mannose receptor that is present on antigenpresenting cells, and this may also lead to the activation of complement-dependent phagocytosis. This technology was previously shown to be effective in stimulating strong cellular immune responses in humans in the context of HIV vaccination, possibly via uptake by and activation of dendritic cells in the skin [78]. By using a heterologous prime-boost strategy consisting of a single intramuscular DNA and a single adjuvanted recombinant protein injection, de Filette et al. [77] showed that $1 \mu \mathrm{g}$ of DNA was sufficient to efficiently prime protective anti-WNV immune responses. The ability of DNA to prime immune cells for the contact with other vaccine antigens such as recombinant proteins is being used more frequently in vaccinology [79] and has delivered powerful immunization strategies against WNV [71,80]. Other approaches that have been tested for the delivery of WNV DNA vaccines include nanopatches that target the plasmid directly to antigen presenting cells in the epidermis [81].

\section{Vector-based vaccines}

Insertion of the WNV E protein with or without the prM protein into a variety of viral vector systems has generated several promising vaccine candidates. The $17 \mathrm{D}$ yellow fever vaccine strain is the basis of the ChimeriVax ${ }^{\mathrm{TM}}$ (SanofiPasteur, Lyon, France) technology. Here, the $\operatorname{pr} M / E$ gene of $\mathrm{YF}$ is replaced by the corresponding sequences of WNV. The resulting chimeric flavivirus is attenuated but remains immunogenic enough to elicit neutralizing antibodies [82]. A ChimeriVax-JEV vaccine was licensed for use in humans and it is one of the most advanced WNV vaccine candidates, having passed Phase II clinical trials [20,83]. A similar vaccine for horses was commercialized but later recalled due to allergic reactions of immunized horses against an excipient in the formulation [84]. Another chimeric flavivirus for WNV vaccination is generated by using an attenuated dengue virus as backbone and swapping the $\mathrm{prM} / \mathrm{E}$ genes as above [85]. Results in animal models and clinical Phase I testing of this vaccine show the induction of robust protective immune responses. Other vector-based systems that were successfully used to immunize animals against WNV include measles virus, 
vesicular stomatitis virus, influenza A virus, lentiviruses, adenovirus and the commercially available veterinary WNV vaccine based on the canarypox virus [22,86-90].

\section{Towards a human vaccine}

A human WNV vaccine has to address a couple of specific issues, which make its development more complex than expected on basis of the promising results obtained with many of the technologies described above.

The human population groups at highest risk for developing WNV disease are the elderly or immunocompromised individuals $[2,91]$. This complicates the use of vaccine candidates that consist of, for example, liveattenuated viruses or live viral vector systems, as these might cause severe risks in the context of an impaired immune system. Inactivated vaccines, recombinant proteins or DNA are clearly advantageous as vaccine antigens in this context. On the other hand, it is well established that protective immunity is harder to achieve in the context of immunosenescence [92]. As a consequence, such vaccines need to be especially immunogenic, which would then favor modified live virus technologies or the use of potent adjuvants with repeated rounds of immunization. The age of the immunized individual has only been addressed by very few of the WNV vaccine studies described so far, mostly very young animals are being used. Ledgerwood et al. [70] found that a DNA vaccine was equally immunogenic in people below or above the age of 50 years. Pinto et al. [93] used aged mice to compare the hydrogen peroxide inactivated whole-virus vaccine with a commercially available formaldehyde-inactivated WNV vaccine. Although the antibody titers elicited with both vaccines were very similar, full protection was only observed with the commercial one. Potential caveats of such comparisons are the different amounts of virus particles in the product and their degree of purification plus the adjuvants used in the different vaccines [93]. Nevertheless, these studies demonstrate the importance of including aged animals in WNV vaccine research in order to specifically address protection in the context of immunosenescence. In addition, it will be a critical issue to establish WNV antibody titers that correlate with the protection of humans from disease, and to integrate these results into the different animal models.
WNV vaccine development is also challenged by the variability of circulating virus strains. This is of special importance in Europe where, in contrast to the Americas, WNV strains belonging to different genetic lineages are co-circulating, sometimes even in the same area [94]. Almost all candidate vaccines have so far been developed based on lineage 1 sequences (such as the NY99 strain), and the question arises whether they also mediate protection against the recently isolated lineage 2 isolates. However, the issue of crossprotection has been addressed in some recent studies, and the results demonstrate that the lineage 1 based vaccines tested indeed show broad crossprotection against lineage 2 viruses $[60,95,96]$. This is in accordance to studies with JEV vaccines, which are based on genotype III but elicit antibodies that crossneutralize other genotypes [97]. Hence, the genetic diversity of WNV does not seem to be that problematic for vaccine development at the moment. On the other hand, the differences of WNV lineages should always be kept in mind, since the situation might be different with future emerging strains.

Another factor complicating the development of a human WNV vaccine is the relatively low incidence of infections combined with the difficulty in clinically testing of the protective efficacy. WNV outbreaks are occurring sporadically and in an unpredictably way. As an example, the focus of the 2012 epidemic in the USA was in Texas with the highest case numbers since 2003. However, in 2013, the incidence was much lower and highest case numbers were counted in California [98]. It is, therefore, difficult to allocate complex and expensive clinical trials to a defined geographical area. As already pointed out by others $[28,82,99]$, these problems need to be discussed jointly by regulatory authorities and the vaccine industry.

\section{Conclusion \& future perspective}

Many obstacles in the development of an efficacious vaccine against WNV were overcome in the last decade. Research efforts have not only delivered the available veterinary vaccines but also technologies that elicit strong protective immune responses in a variety of animal models and humans. To develop the first human WNV vaccine, these technologies need to be transformed into powerful and safe immunogens that can be used in the population groups which are mostly affected by WNV. In addition, solutions 
have to be found for problems due to market size and clinical efficacy testing. It is not in the line of expectation that WNV will lose its threat in near future. On the contrary, the virus is continuing its spread throughout the world and novel genetic variants are arising [9,100]. Therefore, having a

\section{EXECUTIVE SUMMARY}

\section{West Nile virus}

- West Nile virus (WNV) is an emerging mosquito-borne virus which infects birds but can be transmitted to mammals including humans and horses.

- Infections remain asymptomatic or cause mild symptoms in $80 \%$ of the cases.

- Severe neurological disease, such as meningitis or encephalitis, can occur, and older or immunocompromised individual are at highest risk.

- WNV is increasing its distribution in Europe, its neighboring areas and the Americas.

\section{Vaccine-mediated protection from WNV}

- There is no human vaccine available.

- Several veterinary vaccines exist.

- Vaccine-mediated protection from WNV is mostly antibody mediated.

- Key neutralizing epitopes are situated in the $\mathrm{E}$ (envelope) protein of WNV.

Vaccines containing complete WNV particles

- Inactivated vaccines are used to protect horses.

- Novel inactivation procedures yield vaccines with better preserved antigens.

\section{Recombinant protein vaccines}

- Major target of recombinant protein vaccines is the E protein, either as entire ectodomain or as part of subviral particles; also the nonstructural protein 1 is target of protective antibodies.

- The use of different adjuvants has strong effects on immunogenicity and protective capacity of recombinant antigens.

- Strategies to express the E protein in bacteria or insect cells have yielded candidate vaccines in late preclinical and early clinical phases of development.

\section{DNA vaccines}

- A DNA vaccine coding for the prM and E proteins of WNV was licensed for veterinary use and tested in a clinical Phase I trial in humans.

- Several techniques are in preclinical development to successfully deliver DNA vaccines, including microneedles or nanoparticles.

\section{Vector-based vaccines}

- A chimeric yellow fever vaccine virus expressing the prM and E protein of WNV has been developed up to a Phase II clinical trial in humans.

- Several other vector systems, mainly also chimeric for the WNV prM/E proteins, have been established.

\section{Towards a human vaccine}

- Several candidate vaccines for the use in humans have been developed which show effective immunogenicity and display excellent preclinical and clinical safety profiles.

- Levels of neutralizing antibodies that correlate to protection need to be established.

- Clinical development of human WNV vaccines has to take into account issues such as immunosenescence of the at-risk population, low incidence and sporadic occurrence of outbreaks and requires the involvement of both vaccine industry and health authorities. 
human vaccine available is critical for the preparedness to protect the population against this emerging infectious disease.

\section{Acknowledgements}

The authors thank Drs Jenny Reimer, Linda Stertman, Thomas Grunwald and Ernst Verschoor (BPRC, The Netherlands) for helpful comments on the manuscript.

Financial \& competing interests disclosure

SE Magnusson is an employee of Novavax AB and a minority shareholder of Novavax Inc. The authors have no other relevant affiliations or financial involvement with any organization or entity with a financial interest in or financial conflict with the subject matter or materials discussed in the manuscript apart from those disclosed.

No writing assistance was utilized in the production of this manuscript.

\section{Open access}

This work is licensed under the Creative Commons Attribution-NonCommercial 3.0 Unported License. To view a copy of this license, visit http://creativecommons.org/ licenses/by-nc-nd/3.0/

\section{References}

Papers of special note have been highlighted as:

- of interest; $\bullet$ of considerable interest

1 Gubler DJ, Kuno G, Markoff L. Flaviviruses. In: Fields Virology (5th Edition). Knipe DM, Howley PM (Eds). Lippincott Williams and Wilkins, Philadelphia, PA, USA, 1155-1252 (2007).

2 Hayes EB, Sejvar JJ, Zaki SR, Lanciotti RS, Bode AV, Campbell GL. Virology, pathology, and clinical manifestations of West Nile virus disease. Emerg. Infect. Dis. 11, 1174-1179 (2005).

3 Papa A. West Nile virus infections in humans - focus on Greece. J Clin. Virol. 58, 351-353 (2013).

4 Murray KO, Mertens E, Desprès P. West Nile virus and its emergence in the United States of America. Vet. Res. 41, 67 (2010).

5 LaDeau SL, Kilpatrick AM, Marra PP. West Nile virus emergence and large-scale declines of North American bird populations. Nature 447, 710-713 (2007).

6 Lanciotti RS, Ebel GD, Deubel V et al. Complete genome sequences and phylogenetic analysis of West Nile virus strains isolated from the United States, Europe, and the Middle East. Virology 298, 96-105 (2002).

7 Botha EM, Markotter W, Wolfaardt M et al. Genetic determinants of virulence in pathogenic lineage 2 West Nile virus strains. Emerg. Infect. Dis. 14, 222-230 (2008).

8 Papa A, Xanthopoulou K, Gewehr S, Mourelatos S. Detection of West Nile virus lineage 2 in mosquitoes during a human outbreak in Greece. Clin. Microbiol. Infect. 17(8), 1176-1180 (2011).

9 Bakonyi T, Ferenczi E, Erdélyi K et al. Explosive spread of a neuroinvasive lineage 2 West Nile virus in Central Europe, 2008/2009. Vet. Microbiol. 165(1-2), 61-70 (2013).
10 Magurano F, Remoli ME, Baggieri M et al. Circulation of West Nile virus lineage 1 and 2 during an outbreak in Italy. Clin. Microbiol. Infect. 18, 545-547 (2012).

11 Barzon L, Pacenti M, Franchin E et al. The complex epidemiological scenario of West Nile virus in Italy. Int. J. Environ. Res. Public Health. 10, 4669-4689 (2013).

12 Mukhopadhyay S, Kuhn RJ, Rossmann MG. A structural perspective of the flavivirus life cycle. Nat. Rev. Microbiol. 3(1), 13-22 (2005).

13 Chung KM, Thompson BS, Fremont DH, Diamond MS. Antibody recognition of cell surface-associated NS1 triggers Fc-gamma receptor-mediated phagocytosis and clearance of West Nile Virus-infected cells. J. Virol. 81, 9551-9555 (2007).

14 Chung KM, Diamond MS. Defining the levels of secreted non-structural protein NS1 after West Nile virus infection in cell culture and mice. J. Med. Virol. 80(3), 547-556 (2008).

15 Chung KM, Nybakken GE, Thompson BS et al. Antibodies against West Nile Virus nonstructural protein NS1 prevent lethal infection through Fc gamma receptordependent and -independent mechanisms. J. Virol. 80, 1340-1351 (2006).

16 Chung KM, Liszewski MK, Nybakken G et al. West Nile virus nonstructural protein NS1 inhibits complement activation by binding the regulatory protein factor H. Proc. Natl Acad. Sci. USA 103, 19111-19116 (2006).

17 Arjona A, Ledizet M, Anthony K et al. West Nile virus envelope protein inhibits dsRNA-induced innate immune responses. J. Immunol. 179, 8403-8409 (2007).

18 Laurent-Rolle M, Boer EF, Lubick KJ et al. The NS5 protein of the virulent West Nile virus NY99 strain is a potent antagonist of type I interferon-mediated JAK-STAT signaling. J. Virol. 84, 3503-3515 (2010).
19 Halstead SB, Thomas SJ. Japanese Encephalitis: New Options for Active Immunization. Clin. Infect. Dis. 50, 1155-1164 (2010).

20 Ishikawa T, Yamanaka A, Konishi E. A review of successful flavivirus vaccines and the problems with those flaviviruses for which vaccines are not yet available. Vaccine 32 , 1326-1337 (2014).

$21 \mathrm{Ng}$ T, Hathaway D, Jennings N, Champ D, Chiang YW, Chu HJ. Equine vaccine for West Nile virus. Dev. Biol. (Basel) 114, 221-227 (2003).

22 El Garch H, Minke JM, Rehder J et al. A West Nile virus (WNV) recombinant canarypox virus vaccine elicits WNV-specific neutralizing antibodies and cell-mediated immune responses in the horse. Vet. Immunol. Immunopathol. 123, 230-239 (2008).

23 Gardner IA, Wong SJ, Ferraro GL et al. Incidence and effects of West Nile virus infection in vaccinated and unvaccinated horses in California. Vet. Res. 38(1), 109-116 (2007).

24 Iyer AV, Kousoulas KG. A review of vaccine approaches for West Nile virus. Int. J. Environ. Res. Public Health. 10(9), 4200-4223 (2013).

25 Diamond MS, Mehlhop E, Oliphant T, Samuel MA. The host immunologic response to West Nile encephalitis virus. Front. Biosci. 14, 3024-3034 (2009).

26 Lim SM, Koraka P, Osterhaus AD, Martina BE. West Nile virus: immunity and pathogenesis. Viruses 3(6), 811-828 (2011).

27 Netland J, Bevan MJ. CD8 and CD4 T cells in west nile virus immunity and pathogenesis. Viruses 5(10), 2573-2584 (2013).

28 Suthar MS, Diamond MS, Gale M Jr. West Nile virus infection and immunity. Nat. Rev. Microbiol. 11(2), 115-128 (2013).

- Comprehensive review, focusing on innate immune responses 
29 Diamond MS, Shrestha B, Marri A, Mahan D, Engle M. B cells and antibody play critical roles in the immediate defense of disseminated infection by West Nile encephalitis virus. J. Virol. 77, 2578-2586 (2003).

30 Diamond MS, Sitati EM, Friend LD, Higgs S, Shrestha B, Engle M. A critical role for induced $\operatorname{IgM}$ in the protection against West Nile virus infection. J. Exp. Med. 198, 1853-1862 (2003).

31 Oliphant T, Engle M, Nybakken GE et al. Development of a humanized monoclonal antibody with therapeutic potential against West Nile virus. Nat. Med. 11(5), 522-530 (2005).

32 Beigel JH, Nordstrom JL, Pillemer SR et al. Safety and pharmacokinetics of single intravenous dose of MGAWN1, a novel monoclonal antibody to West Nile virus. Antimicrob. Agents Chemother. 54(6), 2431-2436 (2010).

33 Purtha WE, Tedder TF, Johnson S, Bhattacharya D, Diamond MS. Memory B cells, but not long-lived plasma cells, possess antigen specificities for viral escape mutants. J. Exp. Med. 208(13), 2599-2606 (2011).

- First detailed analysis of the basis for vaccine-induced antibody- mediated protection against WNV

34 Mehlhop E, Nelson S, Jost CA et al. Complement protein $\mathrm{Clq}$ reduces the stoichiometric threshold for antibody-mediated neutralization of West Nile virus. Cell Host Microbe 6(4), 381-391 (2009).

35 Shrestha B, Ng T, Chu HJ, Noll M, Diamond MS. The relative contribution of antibody and CD8+ T cells to vaccine immunity against West Nile encephalitis virus. Vaccine 26, 2020-2033 (2008).

36 Wang T, Anderson JF, Magnarelli LA, Wong SJ, Koski RA, Fikrig E. Immunization of mice against West Nile virus with recombinant envelope protein. J. Immunol. 167(9), 5273-5277 (2001).

37 Chabierski S, Makert GR, Kerzhner A et al. Antibody responses in humans infected with newly emerging strains of West Nile Virus in Europe. PLoS ONE 8(6), e66507 (2013).

38 Vázquez S, Guzmán MG, Guillen G et al. Immune response to synthetic peptides of dengue prM protein. Vaccine 20(13-14), 1823-1830 (2002).

39 Beasley DW, Barrett AD. Identification of neutralizing epitopes within structural domain III of the West Nile virus envelope protein. J. Virol. 76, 13097-13100 (2002).

40 Diamond MS, Pierson TC, Fremont DH. The structural immunology of antibody protection against West Nile virus. Immunol. Rev. 225, 212-225 (2008).

41 Oliphant T, Nybakken GE, Austin SK et al. Induction of epitope-specific neutralizing antibodies against West Nile virus. J. Virol. 81, 11828-11839 (2007).

$42 \mathrm{Chu}$ JH, Chiang CC, Ng ML. Immunization of flavivirus West Nile recombinant envelope domain III protein induced specific immune response and protection against West Nile virus infection. J. Immunol. 178(5), 2699-2705 (2007).

43 Martina BE, Koraka P, van den Doel P, van Amerongen G, Rimmelzwaan GF, Osterhaus AD. Immunization with West Nile virus envelope domain III protects mice against lethal infection with homologous and heterologous virus. Vaccine 26, 153-157 (2008).

44 Throsby M, Geuijen C, Goudsmit J et al. Isolation and characterization of human monoclonal antibodies from individuals infected with West Nile Virus. J. Virol. 80, 6982-6992 (2006).

45 Vogt MR, Dowd KA, Engle M et al. Poorly neutralizing cross-reactive antibodies against the fusion loop of West Nile virus envelope protein protect in vivo via Fcgamma receptor and complementdependent effector mechanisms. J. Virol 85, 11567-11580 (2011).

46 Ferguson M, Wood DJ, Minor PD. Antigenic structure of poliovirus in inactivated vaccines. J. Gen. Virol. 74(4), 685-690 (1993).

47 Brown F. Review of accidents caused by incomplete inactivation of viruses. Dev. Biol. Stand. 81, 103-107 (1993).

48 Amanna IJ, Raué HP, Slifka MK. Development of a new hydrogen peroxide-based vaccine platform. Nat. Med. 18(6), 974-979 (2012).

- Presentation of a novel inactivation method with a high potential for vaccines against a variety of infections

49 Yamshchikov G, Borisevich V, Seregin A. An attenuated West Nile prototype virus is highly immunogenic and protects against the deadly NY99 strain: a candidate for live WN vaccine development. Virology 330 (1), 304-312 (2004).

50 Schlick P, Kofler RM, Schittl B et al. Characterization of West Nile virus live vaccine candidates attenuated by capsid deletion mutations. Vaccine 28, 5903-5909 (2010).

51 Widman DG, Ishikawa T, Giavedoni LD et al. Evaluation of RepliVAX WN, a single-cycle flavivirus vaccine, in a nonhuman primate model of West Nile virus infection. Am. J. Trop. Med. Hyg. 82, 1160-1167 (2010).

52 Whiteman MC, Li L, Wicker JA. Development and characterization of non-glycosylated E and NS1 mutant viruses as a potential candidate vaccine for West Nile virus. Vaccine 28(4), 1075-1083 (2010).

53 Wang T, Anderson JF, Magnarelli LA, Wong SJ, Koski RA, Fikrig E. Immunization of Mice Against West Nile Virus with Recombinant Envelope Protein. J. Immunology 167, 5273-5277 (2001).

54 Ledizet M, Kar K, Foellmer HG et al. A recombinant envelope protein vaccine against West Nile virus. Vaccine 23, 3915-3924 (2005).

55 Lieberman MM, Clements DE, Ogata $S$ et al. Preparation and immunogenic properties of a recombinant West Nile subunit vaccine. Vaccine 25(3), 414-423 (2007).

56 Bonafé N, Rininger JA, Chubet RG et al. A recombinant West Nile virus envelope protein vaccine candidate produced in Spodoptera frugiperda expresSF+ cells. Vaccine 27(2), 213-222 (2009).

- Thorough investigation of an insect-cell derived WNV E vaccine candidate studied in several animal species, including horses

57 Watts DM, Tesh RB, Siirin M et al. Efficacy and durability of a recombinant subunit West Nile vaccine candidate in protecting hamsters from West Nile encephalitis. Vaccine 25( 15), 2913-2918 (2007).

58 Lieberman MM, Nerurkar VR, Luo $\mathrm{H}$ et al. Immunogenicity and protective efficacy of a recombinant subunit West Nile virus vaccine in rhesus monkeys. Clin. Vaccine Immunol. 16(9), 1332-1337 (2009).

- Comprehensive study on the protective effect of an adjuvanted recombinant WNV $E$ vaccine in non-human primates, with investigation of the humoral and cellular immune response

59 ClinicalTrials.gov. NCT00707642. http://clinicaltrials.gov/show/NCT00707642

60 Magnusson SE, Karlsson KH, Reimer JM et al. Matrix-M ${ }^{\mathrm{TM}}$ adjuvanted envelope protein vaccine protects against lethal lineage 1 and 2 West Nile virus infection in mice. Vaccine 32(7), 800-808 (2014).

61 Reimer JM, Karlsson KH, Lövgren-Bengtsson K, Magnusson SE, Fuentes A, Stertman L. Matrix-M ${ }^{\mathrm{TM}}$ adjuvant induces local recruitment, activation and maturation of central immune cells in absence of antigen. PLoS ONE 7(7), e 41451 (2012). 
62 Magnusson SE, Reimer JM, Karlsson KH, Lilja L, Bengtsson KL, Stertman L. Immune enhancing properties of the novel Matrix-M ${ }^{\mathrm{TM}}$ adjuvant leads to potentiated immune responses to an influenza vaccine in mice. Vaccine 31(13), 1725-1733 (2013).

63 Cox RJ, Pedersen G, Madhun AS et al. Evaluation of a virosomal $\mathrm{H} 5 \mathrm{~N} 1$ vaccine formulated with Matrix $\mathrm{M}^{\mathrm{TM}}$ adjuvant in a phase I clinical trial. Vaccine 29(45), 8049-8059 (2011).

64 Chu JH, Chiang CC, Ng ML. Immunization of flavivirus West Nile recombinant envelope domain III protein induced specific immune response and protection against West Nile virus infection. J. Immunol. 178(5), 2699-2705 (2007).

65 Zlatkovic J, Stiasny K, Heinz FX. Immunodominance and functional activities of antibody responses to inactivated West Nile virus and recombinant subunit vaccines in mice. J. Virol. 85(5), 1994-2003 (2011).

66 McDonald WF, Huleatt JW, Foellmer HG et al. A West Nile virus recombinant protein vaccine that coactivates innate and adaptive immunity. J. Infect. Dis. 195(11), 1607-1617 (2007).

67 Davis BS, Chang GJ, Cropp B et al. West Nile virus recombinant DNA vaccine protects mouse and horse from virus challenge and expresses in vitro a noninfectious recombinant antigen that can be used in enzyme-linked immunosorbent assays. J. Virol. 75, 4040-4047 (2001).

68 De Filette M, Ulbert S, Diamond M, Sanders NN. Recent progress in West Nile virus diagnosis and vaccination. Vet. Res. 43 , 16 (2012).

69 Martin JE, Pierson TC, Hubka S et al. A West Nile virus DNA vaccine induces neutralizing antibody in healthy adults during a phase 1 clinical trial. J. Infect. Dis. 196, 1732-1740 (2007).

70 Ledgerwood JE, Pierson TC, Hubka SA et al. A West Nile virus DNA vaccine utilizing a modified promoter induces neutralizing antibody in younger and older healthy adults in a phase I clinical trial. J. Infect. Dis. 203(10), 1396-1404 (2011).

71 Schneeweiss A, Chabierski S, Salomo M et al. A DNA vaccine encoding the $\mathrm{E}$ protein of West Nile virus is protective and can be boosted by recombinant domain DIII. Vaccine 29(37), 6352-6357 (2011).

72 Dunn MD, Rossi SL, Carter DM et al. Enhancement of anti-DIII antibodies by the C3d derivative P28 results in lower viral titers and augments protection in mice. Virol. J. 7, 95 (2010).
73 Chang DC, Liu WJ, Anraku I et al. Single-round infectious particles enhance immunogenicity of a DNA vaccine against West Nile virus. Nat. Biotechnol. 26(5), 571-577 (2008).

74 Lechardeur D, Lukacs GL. Intracellular barriers to non-viral gene transfer. Curr. Gene Ther. 2(2), 183-194 (2002).

75 Li L, Saade F, Petrovsky N. The future of human DNA vaccines. J. Biotechnol. 162(2-3), 171-182 (2012).

76 Suh H, Shin J, Kim YC. Microneedle patches for vaccine delivery. Clin. Exp. Vaccine Res. 3(1), 42-49 (2014).

77 De Filette M, Soehle S, Ulbert $S$ et al. Vaccination of mice using the West Nile virus E-protein in a DNA prime-protein boost strategy stimulates cell-mediated immunity and protects mice against a lethal challenge. PLoS ONE 9(2), e87837 (2014).

- Intramuscular application of a DNA vaccine encapsulated in nanoparticles

78 Calarota SA, Weiner DB, Lori F, Lisziewicz J. Induction of HIV-specific memory T-cell responses by topical DermaVir vaccine. Vaccine 25(16), 3070-3074 (2007).

79 Lu S. Heterologous prime-boost vaccination. Curr. Opin. Immunol. 21, 346-351 (2009).

80 Ishikawa T, Takasaki T, Kurane I, Nukuzuma S, Kondo T, Konishi E. Co-immunization with West Nile DNA and inactivated vaccines provides synergistic increases in their immunogenicities in mice. Microbes Infect. 9, 1089-1095 (2007).

81 Prow TW, Chen X, Prow NA et al. Nanopatch-targeted skin vaccination against West Nile Virus and Chikungunya virus in mice. Small 6(16), 1776-1784 (2010).

82 Guy B, Guirakhoo F, Barban V, Higgs S, Monath TP, Lang J. Preclinical and clinical development of YFV 17D-based chimeric vaccines against dengue, West Nile and Japanese encephalitis viruses. Vaccine 28, 632-649 (2010).

83 Biedenbender R, Bevilacqua J, Gregg AM et al. Phase II, randomized, double-blind, placebo-controlled, multicenter study to investigate the immunogenicity and safety of a West Nile virus vaccine in healthy adults. J. Infect. Dis. 203, 75-84 (2011).

84 Brandler S, Tangy F. Vaccines in development against West Nile virus. Viruses 5(10), 2384-2409 (2013).

85 Durbin AP, Wright PF, Cox A et al. The live attenuated chimeric vaccine rWN/DEN4d30 is well-tolerated and immunogenic in healthy flavivirus-naïve adult volunteers. Vaccine 31(48), 5772-5777 (2013).
86 Brandler S, Marianneau P, Loth P et al. Measles vaccine expressing the secreted form of West Nile virus envelope glycoprotein induces protective immunity in squirrel monkeys, a new model of West Nile virus infection. J. Infect. Dis. 206(2), 212-219 (2012).

87 Iyer AV, Pahar B, Boudreaux MJ et al. Recombinant vesicular stomatitis virus-based west Nile vaccine elicits strong humoral and cellular immune responses and protects mice against lethal challenge with the virulent west Nile virus strain LSU-AR01. Vaccine 27(6), 893-903 (2009).

88 Martina BE, van den Doel P, Koraka P et al. A recombinant influenza A virus expressing domain III of West Nile virus induces protective immune responses against influenza and West Nile virus. PLoS ONE 6(4), e18995 (2011).

89 Iglesias MC, Frenkiel MP, Mollier K, Souque P, Despres P, Charneau P. A single immunization with a minute dose of a lentiviral vector-based vaccine is highly effective at eliciting protective humoral immunity against West Nile virus. J. Gene. Med. 8(3), 265-274 (2006).

90 Schepp-Berglind J, Luo M, Wand D et al. Complex adenovirus-mediated expression of West Nile virus C, PreM, E, and NS1 proteins induces both humoral and cellular immune responses. Clin. Vaccine Immunol. 14(9), 1117-1126 (2007).

91 Lindsey NP, Staples JE, Lehman JA, Fischer M. Centers for Disease Control and Prevention (CDC). Surveillance for human West Nile virus disease - United States, 1999-2008. MMWR Surveill. Summ. 59(2), $1-17$ (2010).

92 Boraschi D, Del Giudice G, Dutel C, Ivanoff B, Rappuoli R, Grubeck-Loebenstein B. Ageing and immunity: addressing immune senescence to ensure healthy ageing. Vaccine 28(21), 3627-3631 (2010).

93 Pinto AK, Richner JM, Poore EA et al. A hydrogen peroxide-inactivated virus vaccine elicits humoral and cellular immunity and protects against lethal West Nile virus infection in aged mice. J. Virol. 87(4), 1926-1936 (2013).

94 Magurano F, Remoli ME, Baggieri M et al. Circulation of West Nile virus lineage 1 and 2 during an outbreak in Italy. Clin. Microbiol. Infect. 18, E545-547 (2012).

95 Venter M, van Vuren PJ, Mentoor J, Paweska J, Williams J. Inactivated West Nile Virus (WNV) vaccine, Duvaxyn WNV, protects against a highly neuroinvasive lineage 2 WNV strain in mice. Vaccine 31(37), 3856-3862 (2013). 
96 Minke JM, Siger L, Cupillard L et al. Protection provided by a recombinant ALVAC $^{\circledR}$-WNV vaccine expressing the $\mathrm{prM} / \mathrm{E}$ genes of a lineage 1 strain of WNV against a virulent challenge with a lineage 2 strain. Vaccine 29(28), 4608-4612 (2011).

- First report on cross-protection of WNV genetic lineage 1 based vaccines against a lineage 2 virus.
97 Erra EO, Askling HH, Yoksan S et al. Cross-protective capacity of Japanese encephalitis (JE) vaccines against circulating heterologous JE virus genotypes. Clin. Infect. Dis. 56, 267-270 (2013).

98 Centers for Disease Control and Prevention. West Nile virus. www.cdc.gov
99 Dayan GH, Pugachev K, Bevilacqua J, Lang J, Monath TP. , Preclinical and clinical development of a YFV 17 D-based chimeric vaccine against West Nile virus. Viruses 5(12), 3048-3070 (2013).

100 Vazquez A, Sanchez-Seco MP, Ruiz

$S$ et al. Putative new lineage of west nile virus, Spain. Emerg. Infect. Dis.. 16, 549-552 (2010). 